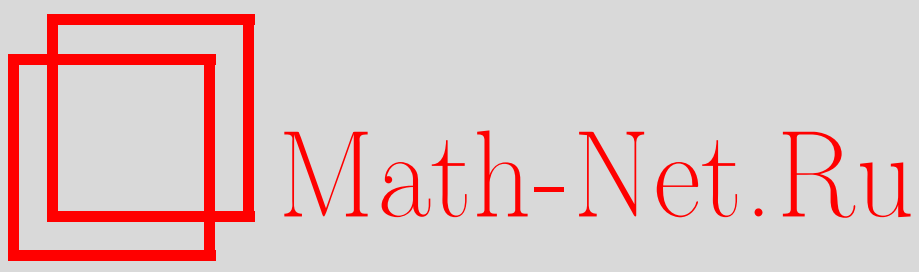

А.М. Шелехов, О фрактальных конструкциях на криволинейной три-ткани, Изв. вузов. Матем., 2019, номер 9, 63-72

DOI: https://doi.org/10.26907/0021-3446-2019-9-63-72

Использование Общероссийского математического портала Math-Net.Ru подразумевает, что вы прочитали и согласны с пользовательским соглашением

http: //www. mathnet.ru/rus/agreement

Параметры загрузки:

IP : 54.172 .240 .79

26 апреля 2023 г., 17:03:07

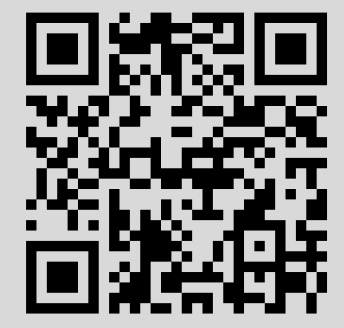


Известия вузов. Математика

2019, № 9, c. 63-72 https://kpfu.ru/science/nauchnye-izdaniya/ivrm

e-mail: izvuz.matem@kpfu.ru

\title{
А.М. ШЕЛЕХОВ
}

\section{О ФРАКТАЛЬНЫХ КОНСТРУКЦИЯХ НА КРИВОЛИНЕЙНОЙ ТРИ-ТКАНИ}

\begin{abstract}
Аннотация. Рассматриваются конфигурации из линий криволинейной три-ткани, которые можно вписать в треугольник, образованный линиями этой ткани. В случае, если вписанная конфигурация является триангулирующей, она порождает в каждом таком треугольнике фрактал. Это обстоятельство позволяет с гладкой функцией двух переменных ассоциировать некоторый фрактал, обобщающий известный треугольник Серпинского. Вводится понятие регулярного фрактала и доказывается, что регулярный фрактал получается только для регулярной три-ткани (обобщение основной теоремы о шестиугольной три-ткани). Найдены фрактальные размерности некоторых регулярных фракталов и сформулированы проблемы, связанные с оценкой фрактальной размерности.
\end{abstract}

Ключевые слова: три-ткань, конфигурация на три-ткани, конфигурация вписанная, конфигурация триангулирующая, фрактал, ассоциированный с три-тканью, обобщенный треугольник Серпинского.

УДК: 514.763

DOI: 10.26907/0021-3446-2019-9-63-72

\section{1. ВПИСАННЫЕ КОНФИГУРАЦИИ НА ТРИ-ТКАНИ}

1. Напомним, что криволинейной три-тканью называют совокупность трех гладких слоений $\lambda_{\alpha}, \alpha=1,2,3$, в некоторой области $D$ евклидовой плоскости. При этом в каждой точке области $D$ проходящие через нее линии три-ткани должны быть попарно трансверсальны. Следуя В.Бляшке, мы рассматриваем три-ткани с точностью до локальных диффеоморфизмов. С помощью подходящего локального диффеоморфизма первые два семейства линий ткани можно выпрямить, так что всякая три-ткань эквивалентна такой три-ткани, у которой первые два семейства образуют декартову сеть. В этом случае линии третьего семейства можно считать линиями уровня некоторой гладкой функции

$$
z=f(x, y) \equiv x \cdot y
$$

которую иногда естественно считать вещественно-аналитической. Переменные $x, y, z$ являются, соответственно, параметрами слоев первого, второго и третьего слоений ткани. Уравнение (1) связывает параметры слоев, проходящих через одну точку $(x, y)$, и называется уравнением ткани. Это уравнение разрешимо (локально) относительно переменных $x$ и $y$, поэтому оно определяет гладкую локальную квазигруппу, которая называется координатной квазигруппой три-ткани $W$. (Бинарная операция $x \cdot y$ называется квазигруппой, если уравнение $z=x \cdot y$ однозначно разрешимо относительно $x$ и $y$. Квазигруппа с единицей называется лупой. Пусть $X, Y, Z$ - три гладких многообразия одинаковой размерности

Поступила в редакцию 15.08.2018, после доработки 15.08.2019. Принята к публикации 19.12.2018 
$r$ (в частности, они могут совпадать). Гладкое отображение $q: X \times Y \rightarrow Z, z=q(x, y)$, называется локальной гладкой $r$-мерной квазигруппой, если оно является локальным диффеоморфизмом a) при фиксированном $x$ и b) при фиксированном $y$. Локальной гладкой $r$-мерной лупой называется $r$-мерное гладкое многообразие $Q$ с фиксированной точкой $e$, ее окрестностью $U$ и функцией $q: U \times U \rightarrow Q$, такой что а) $q$ является локальной гладкой квазигруппой и b) $q(x, e)=q(e, x)=x$ для любого $x$ из $U$. Элемент $e$ называется единицей).

Основным объектом в дифференциально-топологической теории три-тканей являются конфигурации, образованные линиями ткани. Рассмотрим, например, конфигурацию (фигуру) $T$, изображенную на рис. 1 . На нем линии первого, второго и третьего семейств изображены вертикальными, горизонтальными и наклонными линиями соответственно. При этом вертикальные линии отмечены параметрами $x_{\alpha}$, горизонтальные - параметрами $y_{\alpha}$, наклонные - параметрами $z_{\alpha}$. Строится фигура $T$, например, следующим образом. Берем две произвольные линии $x_{1}$ и $y_{1}$ из первого и второго семейств, затем достаточно близко к точке их пересечения проводим наклонные линии $z_{1}$ и $z_{2}$. Через получившиеся точки пересечения проводим вертикальные и горизонтальные линии $x_{2}$ и $y_{2}, x_{3}$ и $y_{3}$, как показано на рис. 1 . Получим точки $A$ и $B$. Построенная конфигурация называется фигурой Томсена или $T$. Если точки $A$ и $B$ лежат на одной линии третьего семейства ткани, то говорят, что фигура $T$ замыкается.

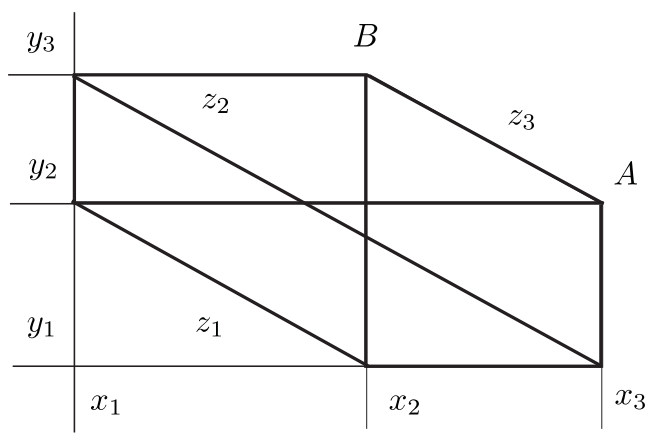

Рис. 1

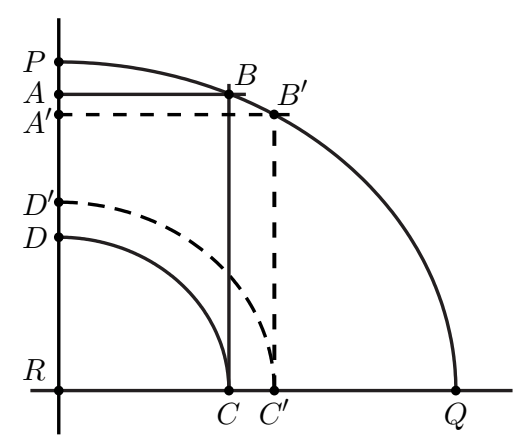

Рис. 2

В частности, если линии $x_{2}, y_{2}$ и $z_{2}$ проходят через одну точку, то фигура $T$ называется шестиугольной конфигурацией или фигурой $H$.

Самая простая три-ткань образована тремя семействами параллельных прямых, она называется параллельной. Эквивалентная ей (локально диффеоморфная) три-ткань называется параллелизуемой или регулярной. Одна из основных теорем теории три-тканей состоит в том, что из замыкания всех достаточно малых фигур $H$ на три-ткани $W$ вытекает ее регулярность ([1], с. 34).

Доказательство этой теоремы основано на следующем факте. Назовем точку $p$ из области определения три-ткани $W$ простой точкой, если в некоторой окрестности этой точки линии три-ткани $W$ слабо изогнуты, т. е. имеют малую интегральную кривизну. Ясно, что у простой точки найдется окрестность, в которой все точки будут простыми. Такие окрестности назовем также простыми. Кроме того, простым будем называть всякий треугольник, образованный в простой окрестности линиями три-ткани $W$. Справедливо

Предложение 1 ([1], с. 33). Пусть $P Q R$ - простой треуголъник, образованный линиями ткани $W$. Тогда в треугольник $P Q R$ можно вписать другой (простой) треугольник, образованный линиями этой ткани. 
Доказательство. Пусть $W$ - произвольная криволинейная три-ткань. Рассмотрим простой треугольник $P Q R$, образованный линиями ткани (рис. 2). Возьмем на одной из сторон треугольника $P Q R$ точку $A$ и впишем в него ломаную $A B C D$ из линий ткани, как показано на рис. 2. Точка $D$, полученная в результате описанной процедуры, не совпадет, вообще говоря, с исходной точкой $A$. Но если точку $A$ двигать к точке $D$ (на рис. 2 она передвинута в положение $A^{\prime}$ ), то, как следует из рис. 2 , точка $D$ будет двигаться ей навстречу. В силу непрерывности функции $D(A)$ у точки $A$ найдется такое положение, при котором $D \equiv A$. В результате получим ситуацию, изображенную на рис. 3.

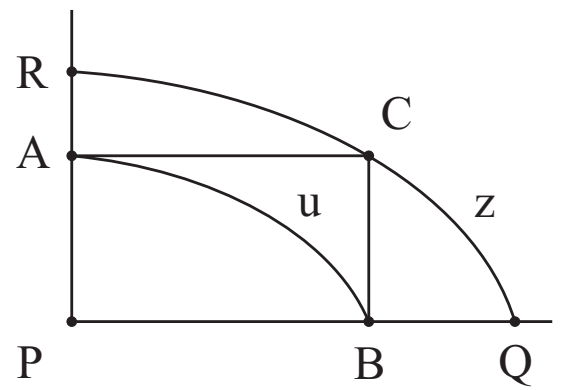

Рис. 3

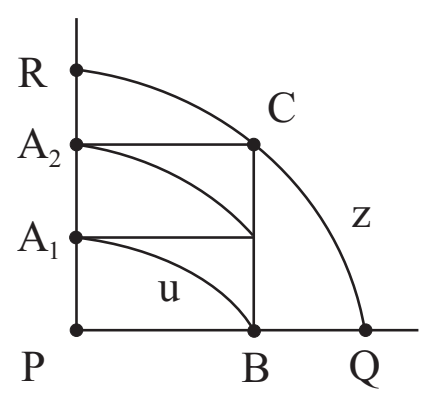

Рис. 4

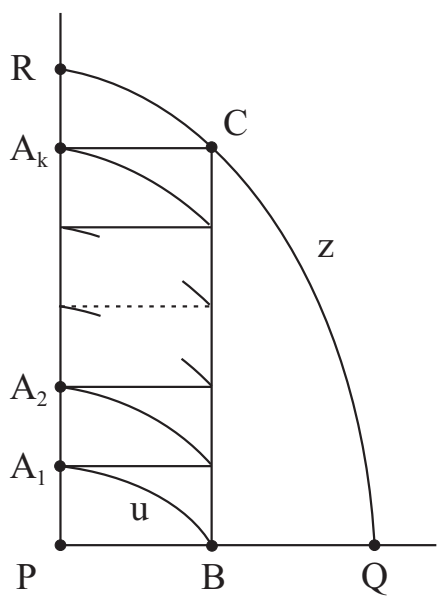

Рис. 5

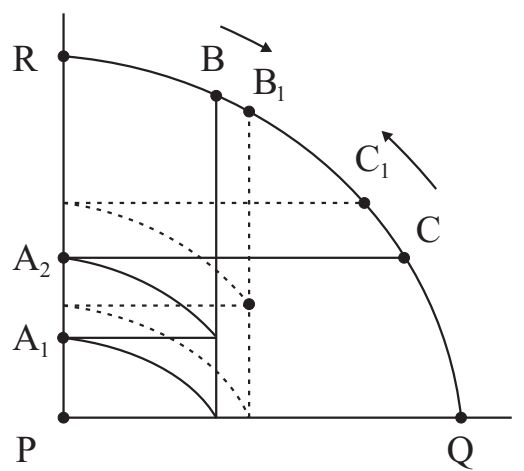

Рис. 6

Аналогично доказывается

Предложение 2. Пусть $P Q R$ - простой треугольник, образованный линиями ткани $W$. Тогда в треугольник $P Q R$ можно вписать конфигурации, изображенные на рис. 4 и 5 , образованные линиями этой ткани.

Доказательство видно из рис. 6.

2. Вписанные конструкции, изображенные на рис. 3-5, можно описать с помощью алгебраической операции, которая называется координатной лупой три-ткани. Пусть $P-$ произвольная простая точка в области определения ткани $W$. Выберем локальные координаты в окрестности точки $P$ так, чтобы горизонтальный и вертикальный слои ткани, 
проходящие через $P$, определялись уравнениями $y=b$ и $x=a$ соответственно. Пусть $u$ и $v$ - слои третьего семейства ткани $W$, достаточно близкие к $P$. Определим в окрестности точки $P(a, b)$ бинарную операцию $u \circ v \equiv \varphi(u, v)$, как показано на рис. 7. Эта операция является локальной гладкой лупой с единицей $e$, так как выполняются равенства $e \circ v=v$ и $u \circ e=u$. Эта лупа называется координатной лупой три-ткани $W$ и обозначается $\ell(P)$ или $\ell(a, b)$.

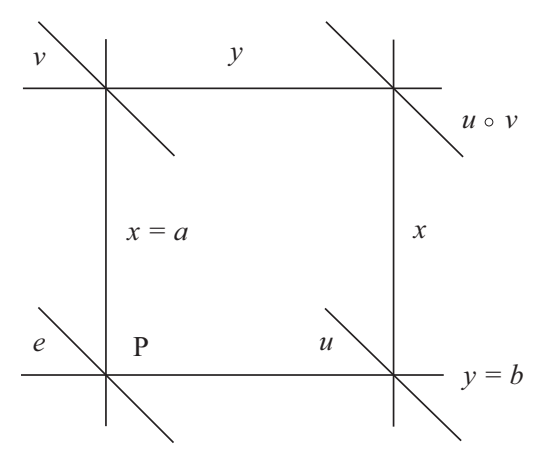

Рис. 7

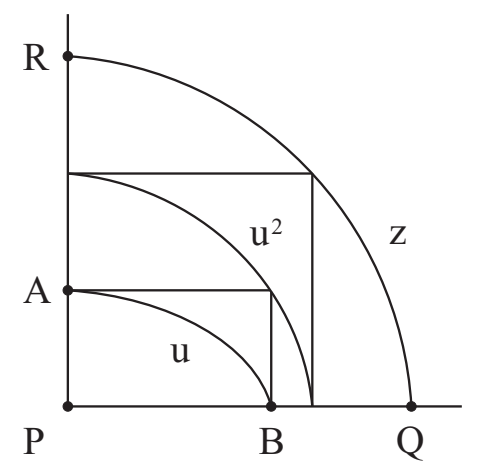

Рис. 8

Обозначим через $u$ следующие слои третьего слоения: $A B$ на рис. $3, A_{1} B$ на рис. 4 и 5 . Пусть, как и выше, символ о обозначает операцию в координатной лупе $\ell(P)$. Непосредственно проверяется

Предложение 3. Переменные $u$ и z, указанные на рис.3-5, связаны следующим образом: на рис. $3 u^{2}=u \circ u=z$, на рис. $4 u \circ u^{2}=z$, на рис. 5

$$
u\left(\circ u\left(\circ \cdots\left(u \circ u^{2}\right) \cdots\right)\right)=z,
$$

причем в последнем произведении и повторяется $k+1$ раз.

Таким образом, каждой из рассмотренных конфигураций, вписанных в простой треугольник три-ткани с "гипотенузой" $z$, соответствует некоторое уравнение вида (2), которое можно назвать уравнением соответствующей конфигурации.

Выше было дано геометрическое доказательство существования вписанных конфигураций. Дадим аналитическое доказательство, основанное на уравнении конфигурации.

Предварительно введем в окрестности точки $P$ стандартную параметризацию. Положим $a=0, b=0$ и $f(0,0)=0$, тогда точка $P$ и слой $e$ (рис. 7 ) получат нулевые координаты. В силу равенств $e \circ v=v$ и $u \circ e=u$ разложение в ряд функции $\varphi(u, v)=u \circ v$ должно иметь вид

$$
\varphi(u, v)=u \circ v=u+v+\eta u v+\lambda u^{2} v+\mu u v^{2}+\cdots,
$$

где в правой части через $u$ и $v$ обозначены параметры линий $u$ и $v$. Поэтому равенство (2) приводит к следующему уравнению на параметр $u:(k+1) u+\cdots=z$. Очевидно, что при фиксированном $z$ это уравнение имеет в окрестности нуля (локально) единственное решение.

Из этих рассуждений получается более общий вывод: уравнение вида $S(u)=z$, где слева стоит произведение элементов координатной лупы с произвольной расстановкой скобок, также будет иметь локально единственное решение, которому будет соответствовать некоторая вписанная конфигурация. Например, уравнению $u^{2} \circ u^{2}=z$ соответствует вписанная конфигурация, изображенная на рис. 8 . 
Вписанные конфигурации будем обозначать через $I C$. В случае, если три-ткань $W$ задана декартовой сетью и линиями уровня функции $z=f(x, y)$, то простой треугольник ограничен линиями $x=a, y=b, f(x, y)=c$, т. е. задается тремя постоянными. Тогда вписанная конфигурация задается набором $I C=(f(x, y), a, b, c, S(u))$ или $I C=(f(x, y)$, $\ell(a, b), S(u)=c)$, где $\ell(a, b)$ - координатная лупа, связанная с точкой $(a, b), S(u)-$ слово от переменной $u$.

\section{2. ФРАКТАЛЬНЫЕ МНОЖЕСТВА, АССОЦИИРОВАННЫЕ С ТРИ-ТКАНЬЮ}

Пусть $P Q R$ - простой треугольник три-ткани $W, I C-$ конфигурация, вписанная в треугольник $P Q R$, причем такая, которая разбивает этот треугольник на более мелкие треугольники. Далее будет видно, что последнее требование можно ослабить. Будем называть такие конфигурации триангулирующими или $t$-конфигурациями. $\mathrm{K} t$-конфигурациям относятся, например, конфигурации, изображенные на рис. $3-5,8$.

Очевидно, что всякая $t$-конфигурация $I C$ порождает внутри треугольника $P Q R$ фрактальное множество с помощью известной процедуры: во все получающиеся треугольники вписываем $t$-конфигурацию $I C$ и затем удаляем часть плоскости, которую она ограничивает. Такой фрактал обозначим $\mathcal{F}=(P Q R, I C)$ или $\mathcal{F}=(P Q R, S(u))$, где $S(u)=c-$ уравнение $t$-конфигурации $I C$. В частности, в самом простом случае, когда $t$-конфигурация $I C$ является треугольником (рис. 3), получается фрактал $\mathcal{F}=\left(P Q R, u^{2}\right)$, который назовем обобщенным треугольником Серпинского (ОТС).

В случае, если три-ткань $W$ задана декартовой сетью и линиями уровня функции $z=$ $f(x, y)$, то указанные выше фрактальные множества будем называть ассоциированными с функцией $f$ и обозначать $\mathcal{F}=(f(x, y), a, b, S(u)=c)$, где $S(u)=c$ - уравнение соответствующей $t$-конфигурации. Таким образом, каждая $t$-конфигурация определяет трехпараметрическое семейство фрактальных множеств, ассочиированных с заданной гладкой функцией двух переменных.

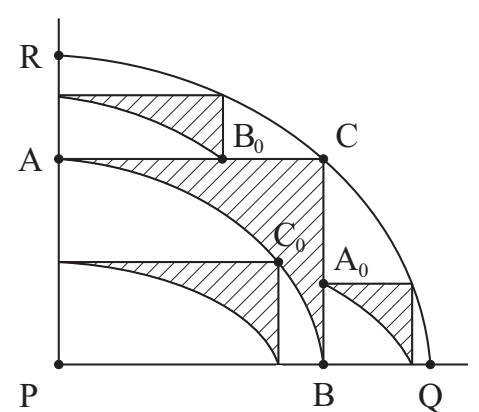

Рис. 9

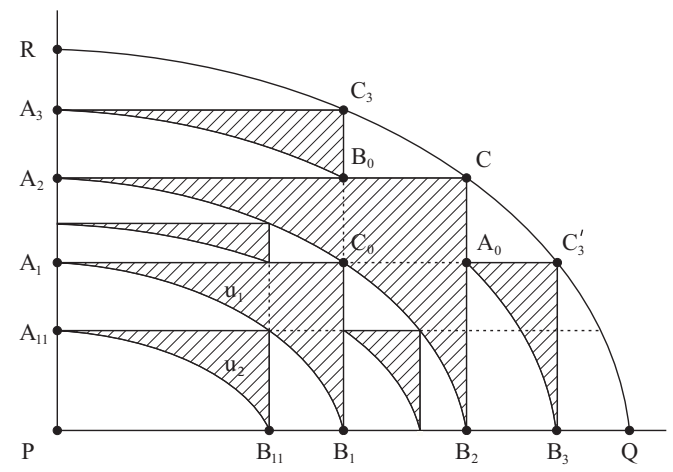

Рис. 10

Рассмотрим рис. 9 , где изображен второй этап получения ОТС. Точки $A_{0}$ и $C_{0}$ не лежат, вообще говоря, на одной горизонтальной линии ткани, а точки $B_{0}$ и $C_{0}$ не лежат, вообще говоря, на одной вертикальной линии этой ткани. В случае, если $A_{0}$ и $C_{0}$ лежат на одной горизонтальной линии ткани, $B_{0}$ и $C_{0}$ лежат на одной вертикальной линии ткани, и аналогичное свойство выполняется при дальнейшем дроблении треугольников, то триангуляцию и соответствующий ОТС будем называть регулярными (рис. 10).

Предложение 4. Если три-ткань $W$ является регулярной, то ОТС является регулярным. 
Доказательство. Действительно, на регулярной три-ткани замыкаются всевозможные шестиугольные фигуры (рис. 1). Отсюда вытекает требуемое свойство.

Докажем обратное утверждение.

Предложение 5. Если для некоторой три-ткани $W$ OTC $\mathcal{F}=\left(f(x, y), a, b, u^{2}=c\right)$, где $f(x, y)$ - вещественно-аналитическал функиия, являетсл регулярным, то и три-ткань W является регулярной.

Лемма. В координатной лупе $\ell(P)$ три-ткани выполняется равенство

$$
\left(u^{2} \circ u\right) \circ u-u \circ\left(u \circ u^{2}\right)=-4 b(P) u^{3}+o_{4},
$$

где $b(P)-$ кривизна ткани $W$ в точке $P$.

Доказательство. Пусть точка $P$ имеет координаты $(0,0)$ и произведение $u \circ v$ в координатной лупе $\ell(P)$ дается формулой (3). Известно ([1], с.57), что разложение (3) можно упростить, а именно, существуют такие параметры слоений ткани, при которых ряд (3) принимает вид

$$
u \circ v=u+v+\lambda u^{2} v+\mu u v^{2}+\cdots .
$$

Непосредственные вычисления дают

$$
\left(u^{2} \circ u\right) \circ u=4 u+(14 \lambda+6 \mu) u^{3}+o_{4}, \quad u \circ\left(u \circ u^{2}\right)=4 u+(6 \lambda+14 \mu) u^{3}+o_{4} .
$$

Отсюда

$$
\left(u^{2} \circ u\right) \circ u-u \circ\left(u \circ u^{2}\right)=8(\lambda-\mu) u^{3}+o_{4} .
$$

С другой стороны, кривизна $b$ три-ткани, заданной уравнением $z=f(x, y)$, вычисляется по формуле ([1], с. 65)

$$
b=\frac{1}{f_{x} f_{y}}\left(\frac{f_{x y y}}{f_{y}}-\frac{f_{x x y}}{f_{x}}+\frac{f_{x x} f_{x y}}{f_{x}^{2}}-\frac{f_{x y} f_{y y}}{f_{y}^{2}}\right) .
$$

Но у нас $f(x, y)=u \circ v$, поэтому, пользуясь (5), находим, что в точке $P(0,0) b=2(\mu-\lambda)$, откуда следует (4).

Доказательство предложения 5.

1. Пусть ОТС $\mathcal{F}=\left(f(x, y), a, b, u^{2}=c\right)$ три-ткани $W$ является регулярным. Рассмотрим рис. 10, на котором изображен второй этап построения ОТС. В силу регулярности возникают вписанные конфигурации $A_{1} A_{3} C_{3} B_{1}$ и $B_{1} A_{1} C_{3}^{\prime} B_{3}$. Обозначим наклонный слой $A_{1} B_{1}=u_{1}$, тогда этим вписанным конфигурациям отвечают в координатной лупе $\ell(P)$ уравнения $\left(u_{1}^{2} \circ u_{1}\right) \circ u_{1}=c$ и $u_{1} \circ\left(u_{1} \circ u_{1}^{2}\right)=c$ соответственно, откуда

$$
\left(u_{1}^{2} \circ u_{1}\right) \circ u_{1}=u_{1} \circ\left(u_{1} \circ u_{1}^{2}\right) .
$$

Выберем, как и выше, координаты так, чтобы точка $P$ имела нулевые координаты, а умножение в координатной лупе $\ell(P)$ задавалось разложением (5). Тогда в силу леммы и соотношения (6) будет выполняться равенство $-4 b(P) u_{1}^{3}+o_{4}=0$, откуда

$$
b(P)=s_{1} u_{1}+s_{2} u_{1}^{2}+\cdots .
$$

На третьем этапе построения фрактала в треугольнике $P A_{1} B_{1}$ получим вписанный треугольник $P A_{11} B_{11}$ с гипотенузой $A_{11} B_{11}=u_{2}$ (рис. 10). В силу определения операции $\circ$ имеем

$$
u_{2}^{2}=u_{1}
$$

Кроме того, из регулярности фрактала будет вытекать равенство, аналогичное (6):

$$
\left(u_{2}^{2} \circ u_{2}\right) \circ u_{2}=u_{2} \circ\left(u_{2} \circ u_{2}^{2}\right) \text {. }
$$


Отсюда получим равенство, аналогичное (7):

$$
b(P)=s_{1} u_{2}+s_{2} u_{2}^{2}+\cdots .
$$

С другой стороны, используя разложение (5), из (8) найдем

$$
u_{2}=\frac{u_{1}}{2}-\frac{1}{16}(\lambda+\mu) u_{1}^{3}+\cdots .
$$

Продолжая процесс, в силу (9) получим бесконечно малую последовательность $u_{1}, u_{2}$, $u_{3}, \ldots\left(u_{n} \rightarrow 0\right)$, причем при любом $n$ будет выполняться равенство

$$
b(P)=s_{1} u_{n}+s_{2} u_{n}^{2}+\cdots .
$$

Отсюда следует $b(P)=0$.

2. Далее заметим, что в процессе построения фрактала на линии $P Q$ будут получаться точки $B_{2}, B_{1}$ и $B_{3}, B_{11}$ и т. д. Эти точки образуют счетное всюду плотное множество. Каждая из них будет вершиной треугольника, в который вписывается ОТС. Проводя для этих треугольников рассуждения как в п. 1, найдем, что кривизна ткани в этих точках равна нулю. Итак, получили, что кривизна обращается в нуль на всюду плотном подмножестве линии $P Q$, откуда, в силу аналитичности функций $f$ и $b$ получаем, что кривизна равна нулю на всей линии $P Q$.

Рассмотрим горизонтальную линию ткани, проходящую, например, через точку $A_{1}$. Проведя для нее аналогичные рассуждения, получим, что кривизна ткани обращается в нуль на всюду плотных подмножествах точек отрезков $A_{1} C_{0}, A_{0} C_{3}^{\prime}$ (рис. 10 ). Отсюда в силу тех же причин следует, что кривизна ткани равна нулю на всей линии $A_{1} C_{3}^{\prime}$.

Теперь рассмотрим горизонтальную линию ткани, проходящую через точку $A_{11}$. При построении фрактала всюду плотные множества вершин треугольников будут возникать на четырех не заштрихованных отрезках этой линии (рис. 10). Аналогичными рассуждениями получаем, что и на этой линии кривизна ткани будет равна нулю.

Далее замечаем, что в процессе построения фрактала точки $A_{2}, A_{1}$ и $A_{3}, A_{11}$ и т.д. образуют счетное всюду плотное множество. Рассмотрим горизонтальную линию ткани, проходящую через какую-либо из них. На ней имеется конечное число заштрихованных отрезков, не принадлежащих фракталу. На оставшихся отрезках вершины вписываемых треугольников образуют счетное всюду плотное множество, поэтому на них кривизна ткани равна нулю. Следовательно, и на всей рассматриваемой горизонтальной линии она равна нулю. В результате получается, что на счетном всюду плотном множестве горизонтальных линий ткани кривизна равна нулю. Поэтому она равна нулю на каждой горизонтальной линии ткани, т. е. в каждой точке треугольника $P Q R$. В силу известной теоремы это означает, что рассматриваемая три-ткань $W$ является регулярной.

Предложения 4 и 5 объединяет

Теорема. OTC $\mathcal{F}=\left(f(x, y), a, b, u^{2}=c\right)$, где $f(x, y)$ - вещественно-аналитическая функиия, является регулярным тогда и только тогда, когда три-ткань $W$, определяемал уравнением $z=f(x, y)$, является регулярной.

Замечание. Теорему можно рассматривать как существенное усиление основной теоремы о шестиугольных три-тканях (см. раздел 1): если на три-ткани замыкаются все достаточно малые шестиугольные фигуры, то эта ткань является регулярной.

Следствие теоремы 1. Всякий регулярный ОТС получается локальным диффеоморфизмом из фрактала, построенного на регулярной три-ткани $z=x+y$. 


\section{3. О РАЗМЕРНОСТИ НЕКОТОРЫХ ФРАКТАЛЬНЫХ МНОЖЕСТВ, АССОЦИИРОВАННЫХ С ТРИ-ТКАНЬЮ}

Найдем размерность фрактала, заданного уравнением $S(u)=c$ в простейшем случае, когда три-ткань является параллельной. Известен следующий факт [2]: пусть фрактал $\mathcal{F}$ получается удалением из множества $M$ последовательности одинаковых конфигураций. Если при удалении конфигурации остается $n$ частей, подобных исходному множеству $M$ с коэффициентами подобия $k_{1}, k_{2}, \ldots, k_{n}$, то фрактальная размерность $s$ фрактала $\mathcal{F}$ находится из уравнения Моргана

$$
k_{1}^{s}+k_{2}^{s}+\cdots+k_{n}^{s}=1 .
$$

$\mathrm{B}$ частности, размерность треугольника Серпинского определяется из равенства $2^{s}=3$.

Пример 1. Параллельная три-ткань $W$ задана декартовой сетью и семейством прямых $x+y=c$, а вписанная конфигурация задается уравнением $u^{2} \circ u^{2}=c$. Она состоит из двух треугольников (рис. 8). Обозначим соответствующий фрактал $\mathcal{F}_{2}$. После удаления вписанной конфигурации остаются три треугольника, подобных исходному треугольнику $P Q R$ с коэффициентом подобия $1 / 4$, и два треугольника, подобных исходному треугольнику $P Q R$ с коэффициентом подобия $1 / 2$. Поэтому фрактальную размерность найдем из формулы

$$
3\left(\frac{1}{4}\right)^{s}+2\left(\frac{1}{2}\right)^{s}=1 .
$$

Отсюда находим $2^{s}=3$, т. е. фрактальная размерность фрактала $\mathcal{F}_{2}$ равна размерности треугольника Серпинского.

Пример 2. Рассмотрим вписанную конфигурацию, обобщающую пример 1, и состоящую из $k$ треугольников: первые два как на рис. 8 , третий вписан в треугольник $P A B$ и т. д. (последовательность треугольников стягивается к точке $P$ ). Если обозначить $u^{2} \circ u^{2}=$ $u^{4}, u^{4} \circ u^{4}=u^{8}$ и т. д., то такая конфигурация задается уравнением $u^{\left(2^{k}\right)}=c$. Обозначим соответствующий фрактал $\mathcal{F}_{k}$. Очевидно, его размерность $s$ находится из равенства

$$
3\left(\frac{1}{2^{k}}\right)^{s}+2\left(\frac{1}{2^{k-1}}\right)^{s}+2\left(\frac{1}{2^{k-2}}\right)^{s}+\ldots+2\left(\frac{1}{2}\right)^{s}=1 .
$$

Положим $2^{-s}=t$, тогда уравнение примет вид

$$
3 t^{k}+2\left(t^{k-1}+t^{k-2}+\cdots+t\right)-1=0
$$

или после преобразований $(3 t-1)\left(t^{k}-1\right)=0$. Таким образом, исходное уравнение имеет единственный вещественный корень $t=1 / 3$, откуда $2^{s}=3$, т. е. опять получаем размерность треугольника Серпинского. Итак, фракталы $\mathcal{F}_{k}$ при любом $k$ имеют одну и ту же фрактальную размерность. Заметим, что этот факт следует из геометрических соображений в пределе у всех фракталов $\mathcal{F}_{k}$ оказываются "выкинутыми" одни и те же треугольники, а различаются они тем, что "выкидывание" происходит при разных $k$ разными группами.

Пример 3. В случае вписанной конфигурации, изображенной на рис. 5, придем к уравнению

$$
(k+1)\left(\frac{1}{k+1}\right)^{s}+\left(\frac{k}{k+1}\right)^{s}=1
$$

или $(k+1)^{s}-k^{s}=k+1$. В частности, при $k=1$ получим треугольник Серпинского, при $k=2$ получим $3^{s}-2^{s}=3$, откуда $s \approx 1.65248$, при $k=3$ имеем $4^{s}-3^{s}=4$, откуда $s \approx 1.68886$. При этом $\lim _{k \rightarrow \infty}=2$, т. е. в пределе получается топологическая размерность, что и следовало ожидать. 
Пример 4. Напоминаем, что рассматривается параллельная три-ткань $W$, заданная декартовой сетью и семейством прямых $x+y=c$. Впишем в треугольник $P Q R t$-конфигурацию как на рис. 5 , затем справа от нее впишем такую же конфигурацию, но один этаж меньше, рядом (правее) - следующую, еще на один этаж меньше и т. д. В силу регулярности ткани полученная триангуляция также будет регулярной в смысле данного выше определения. Всего будет вписано $k+(k-1)+(k-2)+\cdots+1=k(k+1) / 2$ треугольников, после их удаления остается $(k+1)(k+2) / 2$ треугольников, подобных треугольнику $P Q R$ с коэффициентом подобия $1 /(k+1)$. Поэтому в рассматриваемом случае равенство $(10)$ принимает вид

$$
\frac{1}{2}(k+1)(k+2)\left(\frac{1}{k+1}\right)^{s}=1 .
$$

Отсюда

$$
s=\frac{\ln (k+1)(k+2)-\ln 2}{\ln (k+1)} .
$$

В частности, при $k=2,3$ получим $s=1.63093$ и $s=1.66096$ соответственно. При этом $\lim _{k \rightarrow \infty} s=2$, что и должно быть.

Согласно ([3], с.48) фрактальная размерность инвариантна относительно билипшицевых преобразований, в частности, при диффеоморфизмах. При некоторых ограничениях на область определения это утверждение будет справедливо и для локальных диффеоморфизмов. Пусть, например, рассмотренные выше фракталы в примерах 1-4 определены в треугольнике, ограниченном координатными осями и прямой $x+y=1$, и пусть область $D$ есть этот треугольник без начала координат. Тогда, например, отображение $F$ области $D$, заданное уравнениями $F(x, y)=\left(x^{2}, y^{2}\right)$ является диффеоморфизмом на образ. При этом отображении параллельная ткань переходит в регулярную ткань, а образ фрактала будет иметь ту же самую фрактальную размерность, что и прообраз.

В заключение сформулируем некоторые проблемы.

1) Фрактальная размерность фрактала, ассоциированного с функцией $z=f(x, y)$, зависит от формы кривых $f(x, y)=c$, т. е. от их кривизны $k(x, y)$, которая вычисляется по формуле

$$
k=\frac{\left|f_{y}^{2} f_{x x}-2 f_{x} f_{y} f_{x y}+f_{x}^{2} f_{y y}\right|}{\left(f_{x}^{2}+f_{y}^{2}\right)^{\frac{3}{2}}} .
$$

С другой стороны, с три-тканью $W$, заданной декартовой сетью и линиями уровня $f(x, y)=c$, связаны скалярные поля: относительные инварианты кривизна $b(x, y)$ и ее ковариантные производные относительно канонической связности ([1], с. 67), из которых строятся поля абсолютных инвариантов.

Функция $k$ определена во всей области определения три-ткани, а ее обращение в нуль означает, что ткань состоит из прямых (не обязательно параллельных). Обращение в нуль кривизны $b$ означает, что ткань и соответствующий фрактал являются регулярными, т. е. функция $f(x, y)$ имеет вид $f(g(x)+h(y))$.

Задача состоит в том, чтобы (а) оценить фрактальную размерность соответствующего фрактала $\mathcal{F}$ в терминах функций $k(x, y), b(x, y)$ и т. д., (б) выяснить, влияет ли на фрактальную размерность регулярность ткани.

2) Как соотносятся размерности фракталов $\mathcal{F}=(f(x, y), a, b, S(u)=c)$ для одной и той же функции $f(x, y)$ при различных $a, b, c$ ?

Выражаю благодарность А.Е. Миловидову за изготовление рисунков. 


\section{ЛитературА}

[1] Шелехов А.М., Лазарева В.Б., Уткин А.А. Криволинейные три-ткани (Тверь, Тверс. гос. ун-т, 2013).

[2] Mandelbrot B.B. The fractal geometry of nature (W. H. Freeman and Co., San Francisco, 1982).

[3] Kenneth Falconer. Fractal geometry: mathematical foundations and applications (John Wiley and Sons, New York, 1990).

Александр Михайлович Шелехов

Московский педагогический государственный университет, ул. Малая Пироговская, д. 1, стр. 1, г. Москва, 119991, Россия,

e-mail : amshelekhov@rambler.ru

\section{A.M. Shelekhov}

\section{On fractal constructions on curvilinear three-web}

Abstract. We consider configurations of lines of curvilinear three-web that can be inscribed in a triangle formed by the lines of this web. In case the inscribed configuration is triangulating, it generates a fractal in each such triangle. This allows us to associate with smooth function of two variables a certain fractal that generalizes the well-known Sierpiński triangle. We introduce the concept of a regular fractal and prove that a regular fractal is obtained only for a regular three-web (generalization of the basic theorem on hexagonal three-webs). We also find the fractal dimensions of some regular fractals and formulate problems related to fractal dimension.

Keywords: three-web, three-web configuration, inscribed configuration, triangulating configuration, fractal associated with a three-web, generalized Sierpińsky triangle.

Aleksandr Mikhaylovich Shelekhov

Moscow Pedagogical State University, 1 Malaya Pirogovskaya str., bld. 1, Moscow, 119991 Russia,

e-mail : amshelekhov@rambler.ru 